\title{
INTER-COMPARISON OF THE GLOBAL LAND COVER MAPS IN AFRICA SUPPLEMENTED BY SPATIAL ASSOCIATION OF ERRORS
}

\author{
G. Bratic ${ }^{1}$, D. Oxoli ${ }^{1}$, M. A. Brovelli ${ }^{1, *}$ \\ ${ }^{1}$ Politecnico di Milano, Department of Civil and Environmental Engineering, Piazza Leonardo da Vinci 32, 20133 Milan, Italy - \\ (gorica.bratic, daniele.oxoli, maria.brovelli)@polimi.it
}

Commission IV, WG IV/4

KEY WORDS: Land Cover, Inter-comparison, Free and Open Source, Confusion Matrix, Spatial Association

\begin{abstract}
:
Recent advances in Earth Observations supported development of high-resolution land cover (LC) maps on a large-scale. This is an important step forward, especially for developing countries, which experienced problems in the past due to absence of reliable LC information. Nevertheless, increasing number of LC products is imposing additional validation workload to confirm their quality. In this paper inter-comparison of two recent LC products (GlobeLand30 and S2 prototype LC 20m map of Africa) for country of Rwanda in Africa was done. It is a way to facilitate validation by identifying the areas with higher probability of error. Specific approach of comparison of single pixel of one map with multiple pixels of another map provided confusion matrix and sub-pixel agreement table. In this work, accuracy indexes based on the confusion matrix were computed as a measure of similarity between the two maps. Furthermore, Moran's I index was computed for estimation of spatial association of the pixels in disagreement. Also, total disagreement, as well as disagreement of particularly confused classes was visualised to analyse their spatial distribution. The results are showing that similarity of the two maps is about $66 \%$. Disagreements are spatially associated and the most evident in the eastern and north-western part of the area of interest. This coincides also with the distribution of the two most confused classes Wetland and Shrubland. The results delineate areas of inconsistency between the two maps, and therefore areas where careful accuracy analysis are needed.
\end{abstract}

\section{INTRODUCTION}

Land cover (LC) information is well appreciated for a variety of applications, including climate change modeling (Bontemps et al., 2013), natural resource management (Cui et al., 2011) and biodiversity conservation (Kerr, Ostrovsky, 2003). Developing countries, especially those in Africa, were facing problems due to the lack of reliable LC information. For example, prevention of the forest and soil degradation, and mitigation of effects of natural disasters (floods, droughts, and fires) were limited due to scarce LC information (Latham et al., 2002). Recent improvement in Earth Observation programs set up the necessary conditions for improvement in LC maps (Tatem et al., 2008). In fact, many modern LC maps have continental or global extent, resolution up to few meters, and frequent update. Hence, emergence of global land cover maps improved availability of detailed LC information worldwide, including also developing countries.

Validation of LC products is crucial to estimate how well they illustrate reality. Hence, validation confirms if the information contained in LC maps are reliable for practical exploitation. In general, validation is a challenging task. On one hand, depending on the size of the map, it can be computationally intensive. On the other hand, it requires representative reference ("ground truth") data. Selection of reference data sites for global LC products is particularly demanding due to large area.

The aim of this paper was to compare two recent highresolution LC products covering Africa. The accuracy of these products have not been estimated yet, so we wanted to estimate

\footnotetext{
${ }^{*}$ Corresponding author
}

to what extent the information on the two products are consistent/inconsistent. The areas where inconsistency is high could be targets for reference data collection for validation. Intercomparison we have done here can be seen as a preparation for validation. In this way it can be checked if inconsistency is due to error in one of the maps or due to other causes. Analysis were done on the area of Rwanda, as a representative of the developing African countries.

The first map included in the comparison is published by European Space Agency (ESA) Climate Change Initiative (CCI) team. It is called S2 prototype LC $20 \mathrm{~m}$ map of Africa (hereafter CCI Africa Prototype) and it is a map for 2016. It is a representation of African continent at $20 \mathrm{~m}$ resolution using 10 LC classes. Second LC product used here is the GlobeLand30 (GL30) dataset for year 2015. GL30 has resolution of $30 \mathrm{~m}$, and the map for 2015 is the third update of this map. Previously, two maps, for 2000 and 2010 were published. Similarly to the CCI Africa Prototype, GL30 uses 10 classes to describe LC.

A comparison of complete CCI Africa Prototype product with other LC products: Copernicus Global Land Service Land Cover (CGLS-LC100) and Finer Resolution Observation and Monitoring of Global Land Cover for Africa version 2 (FROMGLC-Africa30) was done before (Xu et al., 2019). CGLSLC100 has resolution of $100 \mathrm{~m}$, while FROM-GLC-Africa30 has $30 \mathrm{~m}$ resolution. The approach applied for comparison was to compute accuracy with respect to validation sample set and to compare areas of certain classes with FAO (Food and Agriculture Organization) statistical records at a country level. Then the results for each map were compared among themselves. This study showed that 3 maps have around $40 \%$ of disagree- 
ment.

Datasets in this work were processed in such a way to preserve their resolution as close as possible to the original resolution. This type of processing was reported in one of our previous works (Oxoli et al., 2019). For the purpose of comparison GL30 at $30 \mathrm{~m}$ and CCI Africa Prototype resampled at $10 \mathrm{~m}$ were overlaid. Each pixel of GL30 was compared with 9 corresponding pixels of CCI Africa Prototype. Percentage of the disagreement was assigned to the pixel of GL30.

The maps were analytically compared by means of accuracy indexes based on confusion (error) matrix (Congalton, 2004). However, in this case, instead of accuracy, these indexes are rather expressing similarity or agreement of the two maps. Furthermore, spatial variability and patterns of the disagreement was explored by using spatial association statistics - Moran's I (Moran, 1950).

The results are showing that the overall agreement between the two maps is $66 \%$, which is considered as low agreement. The highest confusion was reported for the classes of Wetland and Shrubland. Furthermore, the spatial association of the confused pixel was strong according to Moran's I.

The paper is structured as follows. In Section 2, the details of the LC dataset used in this work are presented. In Section 3 , methodology of data processing are described. Results are reported and commented in Section 4. Finally, in Section 5 conclusions based on obtained results and ideas for future work are reported.

\section{DATASETS}

Two LC dataset: S2 prototype LC 20m map of Africa and GlobeLand30 were subject of analyses. The analyses were restricted to Rwanda, Africa.

\subsection{GlobeLand30}

GlobeLand 30 (GL30) is a multi-temporal global land cover map produced by National Geomatics Center of China (NGCC). Spatial resolution of this map is $30 \mathrm{~m}$. It was derived from Landsat 7 (NASA, 1999) and HJ-1 (Huan Jing) satellite imagery (NDRCC/SEPA, 2008). The classification was based on Pixel-Object-Knowledge (POK) operational approach (Chen et al., 2015). So far, two versions for years 2000 and 2010 are available under open access licence. GL30 describes globe with 10 generic land cover classes: Cultivated land, Forest, Grassland, Shrubland, Wetland, Water bodies, Tundra, Artificial surfaces, Bareland, Permanent snow and ice. Additional information about classes of GL30 are included in Table 1.

NGCC started production of the GL30 the reference year of 2015. Parts of the GL30 for 2015, covering Horn of Africa (Djibouti, Eritrea, Ethiopia, and Somalia) and several surrounding countries (Burundi, Rwanda, Kenya, Uganda, and Tanzania), are already finished. Dataset will be publicly available when the global map is complete.

In this paper, we analysed the portion of the GL30 for 2015 that coincides with the African country of Rwanda. It has WGS84 reference coordinate system (EPSG:4326) and a resolution of about 30m. Tundra (70), Bareland (90) and Permanent snow and ice (100) LC classes does not exist in Rwanda according to this map.

\begin{tabular}{|c|c|c|}
\hline Code & Class & Definition \\
\hline 10 & $\begin{array}{l}\text { Cultivated } \\
\text { land }\end{array}$ & $\begin{array}{l}\text { Lands used for agriculture, horticulture } \\
\text { and gardens, including paddy fields, ir- } \\
\text { rigated and dry farmland, vegetation and } \\
\text { fruit gardens, etc. }\end{array}$ \\
\hline 20 & Forest & $\begin{array}{l}\text { Lands covered with trees, with vegeta- } \\
\text { tion cover over } 30 \% \text {, including decidu- } \\
\text { ous and coniferous forests, and sparse } \\
\text { woodland with cover } 10-30 \% \text {, etc. }\end{array}$ \\
\hline 30 & Grassland & $\begin{array}{l}\text { Lands covered by natural grass with } \\
\text { cover over } 10 \% \text {, etc. }\end{array}$ \\
\hline 40 & Shrubland & $\begin{array}{l}\text { Lands covered with shrubs with cover } \\
\text { over } 30 \% \text {, including deciduous and } \\
\text { evergreen shrubs, and desert steppe with } \\
\text { cover over } 10 \% \text {, etc. }\end{array}$ \\
\hline 50 & Wetland & $\begin{array}{l}\text { Lands covered with wetland plants and } \\
\text { water bodies, including inland marsh, } \\
\text { lake marsh, river floodplain wetland, } \\
\text { forest/shrub wetland, peat bogs, man- } \\
\text { grove and salt marsh, etc. }\end{array}$ \\
\hline 60 & $\begin{array}{l}\text { Water } \\
\text { bodies }\end{array}$ & $\begin{array}{l}\text { Water bodies in the land area, including } \\
\text { river, lake, reservoir, fish pond, etc. }\end{array}$ \\
\hline 70 & Tundra & $\begin{array}{l}\text { Lands covered by lichen, moss, hardy } \\
\text { perennial herb and shrubs in the polar } \\
\text { regions, including shrub tundra, herb- } \\
\text { aceous tundra, wet tundra and barren } \\
\text { tundra, etc. }\end{array}$ \\
\hline 80 & $\begin{array}{l}\text { Artificial } \\
\text { surfaces }\end{array}$ & $\begin{array}{l}\text { Lands modified by human activities, in- } \\
\text { cluding all kinds of habitation, industrial } \\
\text { and mining area, transportation facilit- } \\
\text { ies, and interior urban green zones and } \\
\text { water bodies, etc. }\end{array}$ \\
\hline 90 & Bareland & $\begin{array}{l}\text { Lands with vegetation cover lower than } \\
10 \% \text {, including desert, sandy fields, } \\
\text { Gobi, bare rocks, saline and alkaline } \\
\text { lands, etc. }\end{array}$ \\
\hline 100 & $\begin{array}{l}\text { Permanent } \\
\text { snow and } \\
\text { ice }\end{array}$ & $\begin{array}{l}\text { Lands covered by permanent snow, gla- } \\
\text { cier and ice cap. }\end{array}$ \\
\hline
\end{tabular}

Table 1. Description of the GL30 classes

Accuracy of GL30 for 2015 have not been assessed yet. Nevertheless, it is expected that accuracy will not deviate too much from accuracy reported for the dataset for 2010 of $80 \%$ (Bratic et al., 2018, Brovelli et al., 2015, Chen et al., 2015).

\subsection{S2 Prototype LC 20m map of Africa}

S2 prototype LC 20m map of Africa (hereafter CCI Africa Prototype) was developed by CCI (Climate Change Initiative) team of ESA (European Space Agency). It was derived by classification of the cloud-free composites of Sentinel-2A observations (ESA, 2015) acquired in 2016. For its derivation, two classification algorithms, Random Forest and Machine Learning were employed in parallel. Each algorithm produced one LC map. The resulting LC maps were combined afterwards by selecting the best representation of the LC class from each of them. Final LC map - CCI Africa Prototype - inherited resolution of $20 \mathrm{~m}$ from the source imagery. It can be downloaded free of charge or visualised on the official web site (ESA CCI Team, 2017). Classification legend (Table 2) was built taking into consideration existing typologies like existing typologies LCCS (Land Cover Classification System) and LCML (Land Cover Meta Language), or legends of other global (e.g. GLC-share, GlobeLand30) and national LC maps (Africover, SERVIR-RMCD). The details related to classes are not available. 


\begin{tabular}{|c|c|}
\hline Code & Class \\
\hline $\mathbf{1}$ & Trees cover areas \\
\hline $\mathbf{2}$ & Shrubs cover areas \\
\hline $\mathbf{3}$ & Grassland \\
\hline $\mathbf{4}$ & Cropland \\
\hline $\mathbf{5}$ & $\begin{array}{c}\text { Vegetation aquatic } \\
\text { or regularly flooded }\end{array}$ \\
\hline $\mathbf{6}$ & $\begin{array}{c}\text { Lichen mosses / } \\
\text { Sparse vegetation }\end{array}$ \\
\hline $\mathbf{7}$ & Bare areas \\
\hline $\mathbf{8}$ & Built up areas \\
\hline $\mathbf{9}$ & Snow and/or ice \\
\hline $\mathbf{1 0}$ & Open water \\
\hline
\end{tabular}

Table 2. CCI Africa Prototype classes

This dataset is characterized as prototype as the official validation of this dataset was not performed. Similarly to GL30, CCI Africa Prototype does not show class 10 - Snow and/or ice in Rwanda.

\section{METHODOLOGY}

Inter-comparison usually requires datasets to be harmonized in term of coordinate reference system, resolution and classification legend. Afterwards datasets are ready for pixel-by-pixel comparison. The methodology we applied does not require the two datasets to have the same resolution. Thus, we kept resolution of the two dataset close to their original resolution. One condition, however, was resolution multiplicity - i.e. one dataset must be multiple integer of the resolution of another dataset. This means that one pixel of lower resolution dataset must be overlapping with certain number of the pixels of higher resolution map. In this way, we were able to compute sub-pixel agreement of the two maps. Preprocessing steps were also influenced by the condition of resolution multiplicity. Complete processing of data, including preprocessing and inter-comparison was done by means of GRASS GIS and its Python Scripting Library (GRASS Development Team, 2018).

\subsection{Data preprocessing}

As initial step, both of the datasets were reprojected to PseudoMercator projected coordinates system (EPSG:3857). After reprojection, the resolution of GL30 was adjusted exactly to $30 \mathrm{~m}$, while resolution of CCI Africa Prototype was set to be $10 \mathrm{~m}$. This means that one pixel of GL30 was spatially coincident with 9 pixels of CCI Africa Prototype. Last preprocessing procedure was devoted to adaption of CCI Africa Prototype classification legend to the legend of GL30. Unfortunately, CCI Africa Prototype classes are defined only by name, and do not contain further description. Therefore, finding a link between classes of the two datasets involved uncertainties. Table 3 is showing reclassification rules we applied for harmonization of classification legends. Preprocessing was done with GRASS GIS functionalities.

\subsection{Sub-pixel comparison}

During the usual inter-comparison, each pixel of one map is compared with a spatially corresponding pixel of another map. The procedure applied here is an exception to the common practice (Oxoli et al., 2019). We compared each pixel of GL30 with 9 pixels of CCI Africa Prototype. Hereafter, we will call it

\begin{tabular}{|c|l|c|l|}
\hline \multicolumn{2}{|c|}{ CCI Africa Prototype } & \multicolumn{2}{c|}{ GlobeLand30 } \\
\hline $\mathbf{1}$ & Tree cover areas & $\mathbf{2 0}$ & Forest \\
\hline $\mathbf{2}$ & Shrubs cover areas & $\mathbf{4 0}$ & Shrubland \\
\hline $\mathbf{3}$ & Grassland & $\mathbf{3 0}$ & Grassland \\
\hline $\mathbf{4}$ & Cropland & $\mathbf{1 0}$ & Cultivated Land \\
\hline $\mathbf{5}$ & $\begin{array}{l}\text { Vegetation aquatic or } \\
\text { regularly flooded }\end{array}$ & $\mathbf{5 0}$ & Wetland \\
\hline $\mathbf{6}$ & $\begin{array}{l}\text { Lichens Mosses / } \\
\text { Sparse vegetation }\end{array}$ & $\mathbf{7 0}$ & Tundra \\
\hline $\mathbf{7}$ & Bare areas & $\mathbf{9 0}$ & Bareland \\
\hline $\mathbf{8}$ & Built up areas & $\mathbf{8 0}$ & Artificial surfaces \\
\hline $\mathbf{9}$ & Snow and/or Ice & $\mathbf{1 0 0}$ & Permanent snow and ice \\
\hline $\mathbf{1 0}$ & Open Water & $\mathbf{6 0}$ & Water bodies \\
\hline
\end{tabular}

Table 3. Link between the classes of CCI Africa Prototype and GL30.

sub-pixel comparison. This procedure is convenient to measure mismatching of GL30 pixel with corresponding pixels of CCI Africa Prototype.

To do inter-comparison, it was needed to create a raster that will keep track of the position of each pixel of GL30. The raster was named ID. It has same size and resolution as GL30. Values of the ID raster were unique ordinal numbers from 0 to 50355358 , which is total number of pixels of GL30. The values were distributed row-wise. To make comparison between GL30 and CCI Africa Prototype, these two rasters and ID raster were overlaid. The example of how one pixel of GL30 is compared with pixels of CCI Africa Prototype is shown on Figure 1a. In this example, the GL30 pixel is 15th in a row, therefore ID value is 15 .

The comparison was done by means of GRASS GIS functionality r.stats (GRASS Development Team, 2003-2019). The output of r.stats is Comma Separated Value (csv) file. The file contains list of all values of CCI Africa Prototype and spatially corresponding values of ID and GL30. This means that values of ID and GL30 are repeating for 9 corresponding pixels of CCI Africa Prototype.

In the next step, raw output of r.stats was modified using Python library Pandas to aggregate the rows with the same ID. This was done by converting csv file into a table. New columns were added for each CCI Africa Prototype class. Then, number of pixels within each class of CCI Africa Prototype with the same ID value was counted and stored in corresponding column (Figure 1b). From this table we derived confusion matrix by summing rows for each GL30 class. Lastly, the aggregated rows were divided by total number of pixels counted in each CCI Africa Prototype class (i.e. it is usually 9, unless ID/GL30 pixel is on the border) (Figure 1c). As a result, we obtained table in which each row reports what is the class of GL30 pixel, as well as to what percent it is in agreement/disagreement with classes in the same area of CCI Africa Prototype. Total disagreement per GL30 pixel was computed and added as the last column of table to have measure of disagreement extent regardless of class. The table is called sub-pixel disagreement table.

Thanks to the ID values, the coordinates of each row were recovered and this table was converted into vector points. This was useful for visual analysis of spatial distribution and association of disagreement.

Regarding spatial association PySal Python library was used for spatial statistics computation. Using this library we computed Global Moran I from sub-pixel disagreement table. 


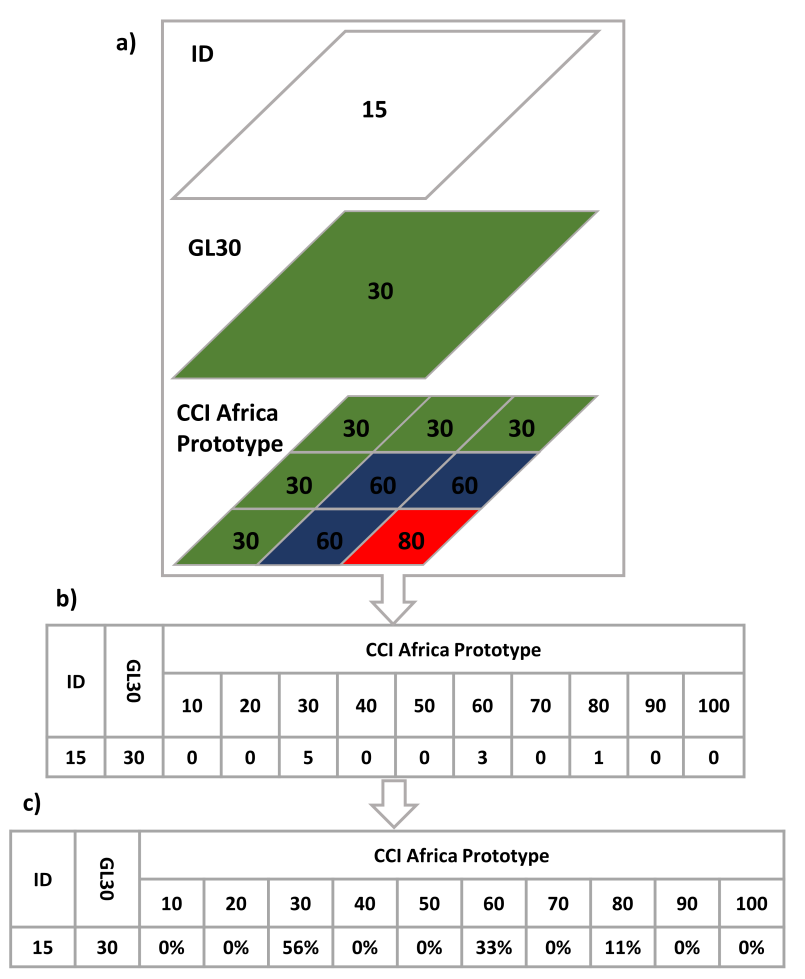

Figure 1. Schematic of the data overlay procedure to compute sub-pixel disagreement on a sample target map pixel $i=15$

Global Moran I weights were distance based k-nearest neighbor weights, where $\mathrm{k}=8$.

\section{RESULTS}

Through data processing we obtained two output useful for inter-comparison: confusion matrix and percentage of confusion of each pixel of GL30 with classes of CCI Africa Prototype. First output was a base to compute overall and per-class agreement/ disagreement indexes, while second one was important for computation of spatial association of disagreement and its distribution.

\subsection{Inter-comparison}

Although the indexes we computed are called accuracy indexes, in this work they serve only to identify similarity between two dataset. We cannot talk about accuracy because none of the datasets has accuracy calculated based on reliable reference data. We can rather point out the agreement or disagreement between the two maps without specifying which of the map is not consistent with the reality.

Table 4 is confusion matrix normalized by columns (i.e. divided by the total number of pixels in a GL30 class). Diagonal elements are analogous to what is usually called Producer's accuracy, when the columns are representing reference map in accuracy assessment (Congalton, 2004). Here, diagonal elements are showing percent of agreement between GL30 and CCI Africa Prototype in each class, measured with respect to total number of pixels per each class of GL30.

According to Table 4, Water (60) and Cropland classes (10) are the most similarly mapped. On the opposite, major disagreement between these two maps is that GL30 does not have pixels classified as Tundra (70) and Bareland (90), while CCI Africa Prototype does. The presence of Tundra on CCI Africa Prototype is a surprise since climate of Rwanda is temperate. Hence, it is most probably error introduced due to harmonization of the classification legends with poor description (Table 3). Absence of Bareland class can not be explained in the same way. Apart from these two classes, the classes with largest disagreement are classes Shrubland (40) and Wetland (50) on GL30, that were confused with Grassland (30) and Forest (20) on CCI Africa Prototype respectively.

\begin{tabular}{|c|l|l|l|l|l|l|l|l|l|l|}
\cline { 2 - 11 } \multicolumn{2}{c|}{} & \multicolumn{10}{c|}{ GlobeLand30 } \\
\cline { 2 - 12 } \multicolumn{2}{c|}{} & $\mathbf{1 0}$ & $\mathbf{2 0}$ & $\mathbf{3 0}$ & $\mathbf{4 0}$ & $\mathbf{5 0}$ & $\mathbf{6 0}$ & $\mathbf{7 0}$ & $\mathbf{8 0}$ & $\mathbf{9 0}$ \\
\hline \multirow{3}{*}{} & $\mathbf{1 0}$ & 79 & 10 & 11 & 6 & 1 & 1 & 0 & 13 & 0 \\
\cline { 2 - 11 } & $\mathbf{2 0}$ & 2 & 48 & 17 & 19 & 60 & 1 & 0 & 6 & 0 \\
\cline { 2 - 11 } & $\mathbf{3 0}$ & 7 & 33 & 50 & 47 & 34 & 2 & 0 & 19 & 0 \\
\cline { 2 - 11 } & $\mathbf{4 0}$ & 11 & 9 & 21 & 27 & 3 & 0 & 0 & 8 & 0 \\
\cline { 2 - 11 } & $\mathbf{5 0}$ & 0 & 0 & 0 & 0 & 1 & 0 & 0 & 0 & 0 \\
\cline { 2 - 10 } & $\mathbf{6 0}$ & 0 & 0 & 0 & 1 & 1 & 95 & 0 & 0 & 0 \\
\cline { 2 - 10 } & $\mathbf{7 0}$ & 0 & 0 & 0 & 0 & 0 & 0 & 0 & 0 & 0 \\
\cline { 2 - 10 } & $\mathbf{8 0}$ & 1 & 0 & 1 & 0 & 0 & 0 & 0 & 54 & 0 \\
\cline { 2 - 11 } & $\mathbf{9 0}$ & 0 & 1 & 0 & 0 & 0 & 0 & 0 & 0 & 0 \\
\hline
\end{tabular}

Table 4. Column-wise normalized confusion matrix [\%]. Numbers in bold represent codes of classes, described in Table 1.

Table 5 is confusion matrix normalized by rows (i.e. divided by the total number of pixels in a CCI Africa Prototype class). Diagonal elements are analogous to what is usually called User's accuracy, when the rows are representing classified map in accuracy assessment (Congalton, 2004). Here, diagonal elements are showing percent of agreement between GL30 and CCI Africa Prototype in each class, measured with respect to total number of pixels per each class of CCI Africa Prototype. The table reports high agreement for classes Cropland (10) and Water (60) which is consistent with the previous table. Confusion, on the opposite, is evident for Shrubland (40) and Wetland (50) classes of CCI Africa Prototype with class Cropland (10) of GL30.

\begin{tabular}{|c|l|l|l|l|l|l|l|l|l|l|}
\cline { 2 - 11 } \multicolumn{2}{c|}{} & \multicolumn{10}{c|}{ GlobeLand30 } \\
\cline { 3 - 12 } \multicolumn{2}{c|}{} & $\mathbf{1 0}$ & $\mathbf{2 0}$ & $\mathbf{3 0}$ & $\mathbf{4 0}$ & $\mathbf{5 0}$ & $\mathbf{6 0}$ & $\mathbf{7 0}$ & $\mathbf{8 0}$ & $\mathbf{9 0}$ \\
\hline \multirow{3}{*}{} & 10 & 91 & 4 & 5 & 0 & 0 & 0 & 0 & 0 & 0 \\
\cline { 2 - 11 } & $\mathbf{2 0}$ & 8 & 58 & 23 & 2 & 9 & 0 & 0 & 0 & 0 \\
\cline { 2 - 11 } & $\mathbf{3 0}$ & 16 & 28 & 48 & 3 & 3 & 0 & 0 & 1 & 0 \\
\cline { 2 - 11 } & $\mathbf{4 0}$ & 48 & 13 & 35 & 3 & 1 & 0 & 0 & 1 & 0 \\
\cline { 2 - 11 } & $\mathbf{5 0}$ & 50 & 6 & 6 & 1 & 17 & 19 & 0 & 2 & 0 \\
\cline { 2 - 10 } & $\mathbf{6 0}$ & 1 & 1 & 0 & 0 & 0 & 98 & 0 & 0 & 0 \\
\cline { 2 - 10 } & $\mathbf{7 0}$ & 30 & 11 & 45 & 5 & 8 & 0 & 0 & 1 & 0 \\
\cline { 2 - 10 } & $\mathbf{8 0}$ & 34 & 4 & 14 & 1 & 0 & 0 & 0 & 47 & 0 \\
\cline { 2 - 10 } & $\mathbf{9 0}$ & 7 & 70 & 10 & 2 & 2 & 8 & 0 & 1 & 0 \\
\hline
\end{tabular}

Table 5. Row-wise normalized confusion matrix [\%]. Numbers in bold represent codes of classes, described in Table 1

Furthermore, index of overall similarity based on the original confusion matrix was computed. It is analogous to Overall accuracy index in accuracy assessment (Congalton, 2004). It accounts for $66 \%$, which means that overall similarity between GL30 and CCI Africa Prototype is not high.

\subsection{Spatial association of pixels in disagreement}

To estimate spatial association, we used sub-pixel disagreement table whose derivation is explained in Section 3.2. Moran's I was used to estimate if the spatial association exists among 
GL30 pixels which are in disagreement with pixels of CCI Africa Prototype. The computed value of Moran's I is around 0.74. This indicates a strong positive spatial association and - in turn - a significant spatial clustering affecting patterns of mismatching pixels. At this preliminary stage of the work, the spatial association is introduced mainly for testing the suitability of the sub-pixel comparison table format to address traditional spatial analysis, such as distance-based statistics. This may provide additional insight into the understanding of disagreement patterns which may be adopted for complementing the outcomes of the maps inter-comparison.

Figure 2 depicts spatial distribution of disagreement of each GL30 pixel with pixels of CCI Africa Prototype. Darker colors are referring to areas with larger disagreements of pixels' classes. This map is showing that disagreement is not equally distributed. Discrepancies are more evident on the eastern part, as well as on the north-west. On the opposite, central and southwestern zones seems to be in agreement.

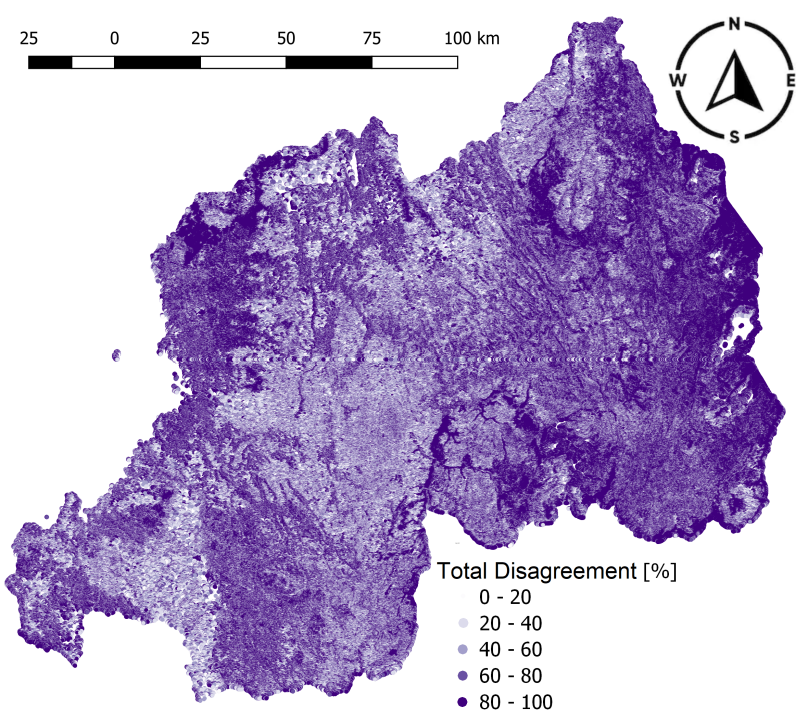

Figure 2. Map of disagreement between GL30 and CCI Africa Prototype

According to the indexes derived from error matrix, the classes of Wetland and Shrubland are the classes with highest confusion. Therefore, we extracted all disagreement values associated to pixels of GL30 which are originally classified as Wetland or Shrubland. Figure 3 is visual representation of disagreement for these two classes.

Green color represent confusion of Shrubland pixels with other classes, while blue color was used for Wetland confusion. The darker the color is, the larger is the magnitude of confusion. The distribution of the Wetland and Shrubland disagreement is matching the areas where disagreement on global level is the highest.

\section{CONCLUSIONS AND FUTURE WORK}

This paper presented procedure of inter-comparison of the two LC products for Africa: GL30 and CCI Africa Prototype. Consistency of the two products is analysed in order to delineate areas with disagreement, and therefore point out where careful accuracy analysis of the maps are needed. Comparison took

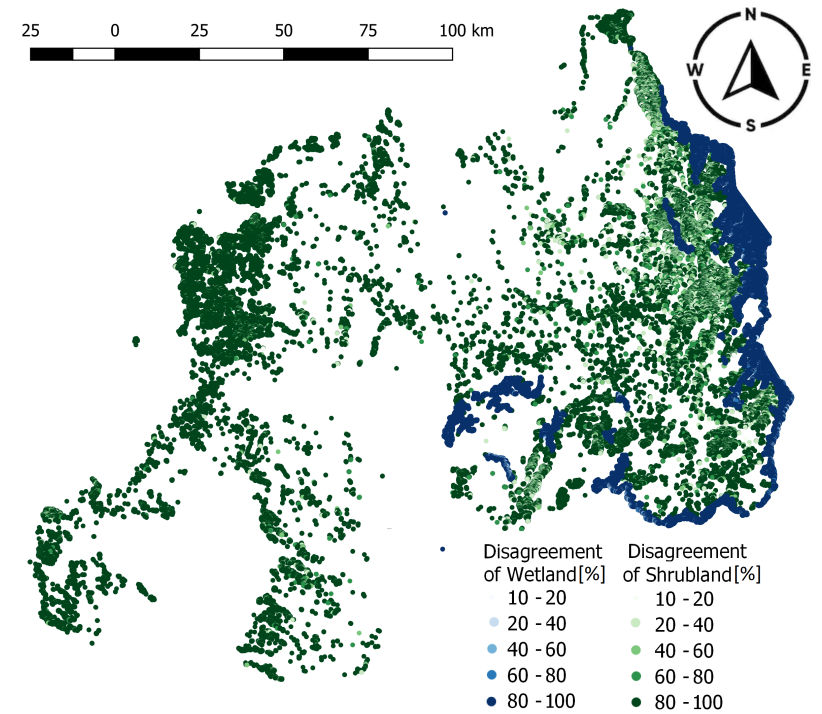

Figure 3. Map of disagreement between Shrubland (green) and Wetland (blue) classes of GL30 with different classes of CCI Africa Prototype.

advantage of accuracy assessment techniques to compute similarity between the maps. Furthermore, disagreement between the two maps was analysed with spatial statistics to estimate spatial association among pixels in disagreement. Finally, mismatching pixels were visualised in order to better understand spatial distribution of disagreement.

Data processing was set up in a specific way to enable comparison of data even if their resolution is not the same. Therefore, the comparison was between single pixel of low-resolution map (GL30), and multiple corresponding pixels of high-resolution map (CCI Africa Prototype). In this way, we were able to estimate sub-pixel thematic agreement/disagreement of each GL30 pixel.

Indexes computed from the error matrix are showing that the agreement between the two maps is rather low - 66\%. Two classes, Wetland and Shrubland have the highest disagreement. Results obtained here are similar to the ones reported by $\mathrm{Xu}$ et al.(2019) although different approaches of comparison were used.

Some of the disagreements are certainly a consequence of adaption of one classification legend to the other in the absence of the complete legend description. This problem is confirmed as Tundra (70) class appears to exist on CCI Africa Prototype after reclassification, although existence of Tundra is not reported on GL30 nor expected in Rwanda. Other sources of disagreement can be ascribed to the different resolution of the two dataset, classification algorithm imperfections, land cover change over time etc.

In the view of the above, these analyses set the ground for the future accuracy assessment of the two datasets. The area of the agreement has higher probability of being accurate, since the two independent datasets are showing same information there. Therefore, focusing on the areas with higher discrepancies can reveal more information about potential errors of the maps.

The introduction of spatial association analysis, besides the numerical results presented in Section 4.2, is considered here as a 
complementary measure to investigate spatial characteristics of disagreements which are not spotted out by e.g. the confusion matrix. Future work will focus on testing spatial association outcomes to support and enrich the traditional inter-comparison analysis.

The work has been carried out using Free and Open Source Software GRASS GIS and Python.

\section{ACKNOWLEDGEMENTS}

We are grateful for support of URBAN-GEO BIG DATA, a Project of National Interest (PRIN) funded by the Italian Ministry of Education, University and Research (MIUR). Moreover, we would like to thank to National Geomatic Center of China (NGCC) and European Space Agency for providing the data.

\section{REFERENCES}

Bontemps, S., Defourny, P., Radoux, J., Van Bogaert, E., Lamarche, C., Achard, F., Mayaux, P., Boettcher, M., Brockmann, C., Kirches, G., Zulkhe, M., Kalogirou, V., Seifert, F. M., Arino, O., 2013. Consistent global land cover maps for climate modelling communities: Current achievements of the ESA' Land Cover CCI. ESA Living Planet Symposium, ESA Special Publication, 722, 62.

Bratic, G., Brovelli, M. A., Molinari, M. E., 2018. A free and open source tool to assess the accuracy of land cover maps: implementation and application to Lombardy Region (Italy). ISPRS - International Archives of the Photogrammetry, Remote Sensing and Spatial Information Sciences, XLII-3, 87-92.

Brovelli, M. A., Molinari, M. E., Hussein, E., Chen, J., Li, R., 2015. The first comprehensive accuracy assessment of GlobeLand30 at a national level: methodology and results. Remote Sensing, 7(4), 4191-4212.

Chen, J., Chen, J., Liao, A., Cao, X., Chen, L., Chen, X., He, C., Han, G., Peng, S., Lu, M., Zhang, W., Tong, X., Mills, J., 2015. Global land cover mapping at $30 \mathrm{~m}$ resolution: A POKbased operational approach. ISPRS Journal of Photogrammetry and Remote Sensing, 103, 7-27.

Congalton, R., 2004. Putting the map back in map accuracy assessment. Remote Sensing and GIS Accuracy Assessment, CRC Press, 1-11.

Cui, G., Lee, W.-K., Kwak, D.-A., Choi, S., Park, T., Lee, J., 2011. Desertification monitoring by LANDSAT TM satellite imagery. Forest Science and Technology, 7(3), 110-116.

ESA, 2015. Sentinel 2. https://sentinel.esa.int/web/sentinel/ missions/sentinel-2. [Online; accessed 19-April-2019].

ESA CCI Team, 2017. S2 prototype LC map at $20 \mathrm{~m}$ of Africa 2016. http://2016africalandcover20m.esrin.esa.int/. [Online; accessed 23-April-2019].

GRASS Development Team, 2003-2019. GRASS GIS 7.7.dev Reference Manual. https://grass.osgeo.org/grass77/manuals/r. stats.html. [Online; accessed 12-April-2019].

GRASS Development Team, 2018. GRASS Python Scripting Library. https://grasswiki.osgeo.org/wiki/GRASS_and_Python. [Online; accessed 24-April-2019].
Kerr, J. T., Ostrovsky, M., 2003. From space to species: ecological applications for remote sensing. Trends in Ecology \& Evolution, 18(6), 299-305.

Latham, J. S., He, C., Alinovi, L., DiGregorio, A., Kalensky, Z., 2002. FAO methodologies for land cover classification and mapping. Linking People, Place, and Policy, Springer US, 283316.

Moran, P. A. P., 1950. Notes on continuous stochastic phenomena. Biometrika, 37(1/2), 17.

NASA, 1999. Landsat 7. https://landsat.gsfc.nasa.gov/ landsat-7/. [Online; accessed 19-April-2019].

NDRCC/SEPA, 2008. Huan Jing (HJ-1). https://earth.esa.int/ web/eoportal/satellite-missions/h/hj-1. [Online; accessed 19April-2019].

Oxoli, D., Bratic, G., Wu, H., Brovelli, M. A., 2019. Extending accuracy assessment procedures of global coverage land cover maps through spatial association analysis. ISPRS - International Archives of the Photogrammetry, Remote Sensing and Spatial Information Sciences, XLII-2/W13, 1601-1607.

Tatem, A., Goetz, S., Hay, S., 2008. Fifty Years of Earthobservation Satellites. American Scientist, 96(5), 390.

Xu, Y., Yu, L., Feng, D., Peng, D., Li, C., Huang, X., Lu, H., Gong, P., 2019. Comparisons of three recent moderate resolution African land cover datasets: CGLS-LC100, ESA-S2LC20, and FROM-GLC-Africa30. International Journal of Remote Sensing, 40(16), 6185-6202. 